\title{
Age-related modifications in rat cardiac phosphoglycerate kinase. Rejuvenation of the old enzyme by unfolding-refolding
}

\author{
Alison Zúñiga and Ari Gafni \\ Institute of Gerontology and Department of Biological Chemistry, University of Michigan, Ann Arbor, MI (U.S.A.)
}

(Received 28 January 1988)

Key words: Enzyme aging; Enzyme folding; Aging; (Rat heart)

The occurrence of age-related modifications in functional and structural properties of several enzymes has been documented; however, the molecular basis of this phenomenon is still mostly unexplained. In the present work a comparative study of phosphoglycerate kinase preparations isolated from hearts of young and old rats was undertaken. Marked age-related effects were revealed in the heat-inactivation kinetics of the enzyme, similar to the ones previously found in purified muscle phosphoglycerate kinase. In view of the previously reported failure of immunotitration to distinguish between phosphoglycerate kinase forms in crude heart extracts from young and old rats, it appears likely that the modifications in old rat heart phosphoglycerate kinase are in a domain which is not involved in antibody binding, and may be localized in the interior of the enzyme. These age-related modifications were completely relieved by extensive unfolding of the enzyme in $2 \mathrm{M}$ guanidine hydrochloride, followed by enzyme reactivation upon dilution of the denaturant. The refolding products of young and old enzymes displayed identical heat-inactivation kinetics as native young phosphoglycerate kinase. It is concluded that the age-related alterations in rat cardiac phosphoglycerate kinase, like those found in the muscle enzyme, are purely conformational and hence develop postsynthetically.

\section{Introduction}

A large volume of experimental results has been accumulated recently showing that a considerable number of enzymes isolated from old animals are altered compared with the same enzymes purified from young animals [1-6]. The age-related modifications may involve changes in the specific enzymatic activity [7], heat stability

\footnotetext{
Abbreviations: PGK, phosphoglycerate kinase (EC 2.7.2.3); Mops, 4-morpholinepropanesulfonic acid.

Correspondence: A. Gafni, Institute of Gerontology and Department of Biological Chemistry, University of Michigan, Ann Arbor, MI 48109, U.S.A.
}

[8], substrate or coenzyme binding patterns [9], spectroscopic properties [10], etc. A hypothesis to explain the occurrence of such changes in enzymes was proposed by Reiss and Rothstein [11] and postulates slower protein turnover in the old tissue leading to longer average dwell times of enzyme molecules in the cell, hence providing more time for them to become modified. The nature of the modifications obviously depends on the particular enzyme; however, the bulk of the data hitherto obtained supports the idea that the aging effects result from conformational modifications without covalent changes [12].

One of the best characterized old enzymes is phosphoglycerate kinase (ATP : 3-phospho-Dglycerate 1-phosphotransferase, EC 2.7.2.3) (PGK). In a series of studies by Rothstein and his 
co-workers, PGK was found to become modified in muscle [13], liver [14] and brain tissues [15] of old rats. While the specific activity in all three cases did not depend on the animal's age, other properties did - in particular, the heat stability of the enzyme was found to increase in old age. Subsequent attempts to reveal covalent modifications in old PGK by isoelectric focusing and by HPLC of proteolytic fragments of the enzyme have failed, and it was concluded that young and old PGKs are conformational isomers [16]. Direct, positive proof for this hypothesis was recently provided by us [17]. The experimental approach taken was based on extensive unfolding of the enzyme in $2 \mathrm{M}$ guanidine- $\mathrm{HCl}$ followed by refolding under mild conditions. Old PGK, thus treated, was found to become identical to the young enzyme in its heat inactivation patterns under various conditions. Since the unfolding-refolding protocol is very unlikely to introduce covalent modifications in the enzyme, these experiments unequivocally demonstrate that the age-related modifications in rat muscle PGK are purely conformational.

Comparative studies of the aging of an enzyme in various organs are of great interest due to the possible existence of multiple isozymes with tissue-dependent distribution and different aging patterns. In addition, the environment provided by different organs may vary, leading to differences in the development and expression of the aging effects in each isozymic form. Such tissuedependent aging effects have indeed been documented. Thus, Rothstein and his co-workers [14] found that the age-related differences between young and old liver PGKs were larger than the corresponding differences in muscle PGK. In addition, while immunotitration of PGK in tissue homogenates revealed that significant age-related differences exist between the enzyme in young and old muscle, brain and liver, the results in kidney and heart were inconclusive [13].

In the present study we demonstrate that rat heart PGK shows pronounced age-related effects, similar (and even larger) to those present in the skeletal muscle and liver enzymes. Moreover, when young and old heart PGKs are unfolded in guanidine- $\mathrm{HCl}$ and subsequently allowed to refold, the reactivation kinetics as well as the re- folded products are strikingly similar. We thus conclude that, as in muscle, young and old heart PGKs are conformational isomers.

\section{Materials and Methods}

Animals. Sprague-Dawley rats were purchased from Harlan Sprague-Dawley, and fed ad libitum with Purina Laboratory Chow. Young animals were 2-4 months of age, while old animals were 28-30 months old. Animals were killed by use of a guillotine, the hearts were removed and washed with $20 \mathrm{mM}$ Tris- $\mathrm{HCl}$ buffer $(\mathrm{pH} \mathrm{7.0)/10} \mathrm{mM}$ EDTA/10 mM 2-mercaptoethanol. Tissue not immediately used was stored at $-80^{\circ} \mathrm{C}$. Enzyme samples purified from fresh or frozen tissue display identical physical and enzymatic properties.

Enzyme isolation and purification. The procedure used in the purification of heart PGK was based on the method described by Sharma et al. [13] for the skeletal muscle enzyme, with some modifications. The heart tissue was washed twice with $20 \mathrm{mM}$ Tris-Mops buffer (pH 7.0)/10 mM sodium EDTA/10 mM 2-mercaptoethanol. The enzyme was extracted by homogenizing the tissue with a 4-fold excess of the same buffer and collecting the supernatant following centrifugation. Ammonium sulfate fractionation was then done by removing the protein precipitate formed at $50 \%$ saturation and bringing the ammonium sulfate concentration in the supernatant to $95 \%$, at which the PGK was fully precipitated. The proteir pellet was dissolved in a minimal volume of $5 \mathrm{mM}$ Tris-Mops buffer (pH 6.7)/10 mM 2-mercaptoethanol $/ 1 \mathrm{mM}$ EDTA, and dialyzed for $6 \mathrm{~h}$ against the same buffer with two buffer changes. The slight turbidity which developed in the enzyme solution during dialysis was removed by centrifugation and the supernatant was applied to a CM-52 column $(2.5 \times 9.0 \mathrm{~cm}$ for $10 \mathrm{~g}$ of tissue) which had been previously equilibrated with $5 \mathrm{mM}$ Tris-Mops (pH 6.7)/10 mM 2-mercaptoethanol/1 mM sodium EDTA. The column was washed with 60 $\mathrm{ml}$ of the same buffer and 2-ml fractions were collected. The PGK was then eluted using a $5 \mathrm{mM}$ D-3-phosphoglycerate solution in the buffer mentioned above. The total protein concentration and the PGK activity were monitored and all fractions containing more than 10 units of activity were 
pooled. Enzyme not being used immediately (i.e., within a few hours) was precipitated by adding ammonium sulfate to $95 \%$ saturation and stored at $4^{\circ} \mathrm{C}$

Protein and enzyme activity assays. Protein concentrations were determined by the method of Bradford [18] using Fraction $V$ bovine serum albumin (Sigma) as the standard. PGK activity was assayed at $25^{\circ} \mathrm{C}$ as recently described [17].

Unfolding and refolding of young and old PGKs. These experiments were done using freshly prepared or ammonium-sulfate-precipitated enzyme samples. In the latter case, excess salt was removed by dialysis ( $4 \mathrm{~h}$ against 100 -fold excess of buffer). The buffer used was either $5 \mathrm{mM}$ TrisMops/1 mM sodium EDTA/10 mM 2mercaptoethanol (pH 6.7) or $100 \mathrm{mM}$ sodium phosphate/10 mM sodium EDTA/10 mM 2mercaptoethanol ( $\mathrm{pH}$ 7.5). Denaturation was achieved by adding to the PGK solution an appropriate volume of a $6 \mathrm{M}$ guanidine- $\mathrm{HCl}$ solution (in water) to make the final concentration of the denaturant $2 \mathrm{M}$, and incubating this mixture for 12-18 $\mathrm{h}$ at $4^{\circ} \mathrm{C}$. Reactivation was achieved by diluting the solution of denatured enzyme into a 10-fold excess of denaturant-free buffer and incubating this solution at $4^{\circ} \mathrm{C}$. Aliquots were taken out at desired intervals and assayed for enzymatic activity. In early experiments enzyme samples designed to serve as controls were treated in exactly the same manner as described above except that pure water, rather than $6 \mathrm{M}$ guanidine- $\mathrm{HCl}$, was mixed with the enzyme solution. We later found that young PGK becomes modified when incubated under these conditions; hence, in most experiments reported here, fresh enzyme (dissolved from its ammonium sulfate precipitate and dialyzed for $4 \mathrm{~h}$ ) was used as the control.

Heat-inactivation experiments. Native young and old forms of PGK, as well as the denatured-renatured forms, were heat-inactivated by adding the enzyme solution into buffer (preheated to the desired temperature) to make the final concentration of enzyme $30 \mu \mathrm{g} / \mathrm{ml}$. Two buffer systems were used: $70 \mathrm{mM}$ Tris-acetate (pH 7.3)/35 $\mathrm{mM}$ $\mathrm{MgCl}_{2} / 10 \mathrm{mM}$ sodium EDTA/10 mM 2mercaptoethanol; or $100 \mathrm{mM}$ sodium phosphate (pH 7.5)/10 mM sodium EDTA/10 mM 2mercaptoethanol. To follow the inactivation rates,
$0.1 \mathrm{ml}$ aliquots were taken out at various times, cooled rapidly on ice and assayed for PGK activity.

\section{Results}

The heat-inactivation kinetics of young and old rat heart PGKs and $44^{\circ} \mathrm{C}$ are presented in Fig. 1. Similar to previous findings with muscle, liver and brain PGKs [2,12], the heat-inactivation rates of the heart enzyme are clearly age-dependent, the old enzyme being more stable than its young counterpart. The differences between the inactivation rates of young and old heart PGKs reported here are considerably larger than the ones found under similar conditions for the skeletal muscle enzyme [13]. This observation is of great interest, since immunotitration experiments revealed only minor differences between PGKs in homogenates from young and old rat hearts [13], indicating that this enzyme may be unaffected by aging. Our present results clearly show that heart PGK does age. Possible reasons for the failure of immunotitration as an indicator of PGK status will be considered in the Discussion.

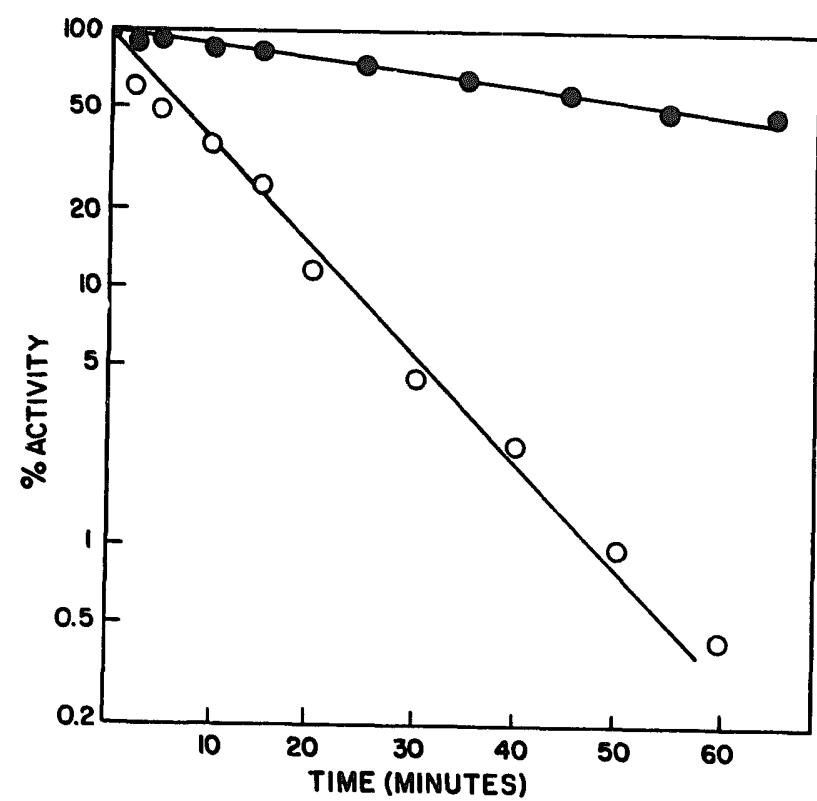

Fig. 1. Heat inactivation of young $(0)$ and old $(\odot)$ rat heart PGKs in $70 \mathrm{mM}$ Tris-acetate buffer (pH 7.3)/35 mM $\mathrm{MgCl}_{2} / 10 \mathrm{mM}$ sodium EDTA/10 mM 2-mercaptoethanol. The concentration of enzyme was $30 \mu \mathrm{g} / \mathrm{ml}$ in both cases and the temperature was $44^{\circ} \mathrm{C}$. 


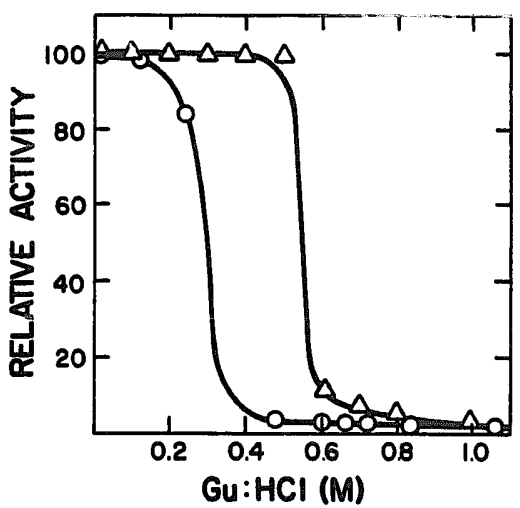

Fig. 2. Inactivation of heart $(0)$ and muscle $(\Delta)$ PGKs from young rats by guanidine- $\mathrm{HCl}$. Each enzyme $(30 \mu \mathrm{g} / \mathrm{ml})$ was incubated in a medium containing $5 \mathrm{mM}$ Tris-Mops buffer (pH 6.7), $10 \mathrm{mM}$ 2-mercaptoethanol, $1 \mathrm{mM}$ EDTA and the indicated concentration of denaturant, at $4^{\circ} \mathrm{C}$ for $18 \mathrm{~h}$. The remaining activity was subsequently determined.

The heat-inactivation of young PGK presented in Fig. 1 obeys first-order kinetics down to less than $1 \%$ of the original activity, indicating that the enzyme is homogeneous. More significantly, the inactivation of the old enzyme does not reveal a fast component and thus demonstrates that old PGK is not contaminated with the young form and is rather homogeneous.

\section{Unfolding-refolding experiments}

When solutions of PGK are incubated with increasing concentrations of guanidine- $\mathrm{HCl}$ for long enough periods of time to ensure that equilibrium is attained (i.e., $12 \mathrm{~h}$ or more), the residual activities decrease as shown in Fig. 2, which compares rat muscle and heart PGKs. The cardiac enzyme is clearly more labile and is fully inactivated at $0.5 \mathrm{M}$ guanidine- $\mathrm{HCl}$, while muscle PGK is still fully active at this concentration of denaturant. This behavior correlates well with the enhanced heat lability of heart PGK (compared with the muscle enzyme) and with the tendency of young heart PGK to become altered in vitro relatively very quickly to a form which resembles old PGK. The kinetics of unfolding of PGK in $2 \mathrm{M}$ guanidine- $\mathrm{HCl}$, as followed through the loss of enzyme activity, are presented in Fig. 3. The activity of PGK was found to decline rapidly to a value of about $3 \%$ of the original activity, which persists for a prolonged time (see Fig .3). This apparent residual activity is an artefact and is due to the presence of PGK contamination in the GPDH used in the assay (Sigma, rabbit muscle). When this residual activity is subtracted from the observed activities at various times and the corrected values plotted on a $\log$ scale vs. time, a linear relationship is observed (Fig. 3, inset), showing that the inactivation reaction is first-order, i.e., involves a transition between two detectable states only. It is pertinent to note that the rapid inactivation of $\mathrm{PGK}$ in $2 \mathrm{M}$ guanidine- $\mathrm{HCl}$ shown in Fig. 3 does not reflect a complete unfolding of the enzyme but rather a transition to an (inactive) unfolding intermediate. Indeed, when PGK denaturation is followed by monitoring changes in the intrinsic protein fluorescence, the process does not reach completion after $1 \mathrm{~h}$ incubation at room temperature (Brown and Gafni, unpublished results). To ensure full denaturation of young and old PGKs (prior to their refolding), we therefore incubated their solutions in $2 \mathrm{M}$ guanidine- $\mathrm{HCl}$ for $12-18 \mathrm{~h}$ at $4^{\circ} \mathrm{C}$.

\section{Refolding of young and old PGKs}

These were monitored by the reactivation of the unfolded enzymes following a 10 -fold dilution of their solutions in guanidine- $\mathrm{HCl}$ into de-

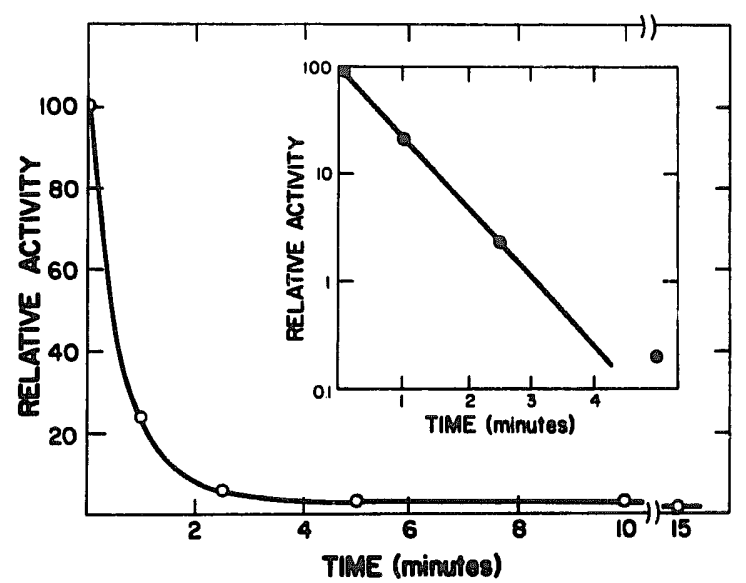

Fig. 3. Kinetics of the inactivation of young rat heart PGK in 2 M guanidine- $\mathrm{HCl} .200 \mu \mathrm{l}$ of PGK solution in $100 \mathrm{mM}$ sodium phosphate buffer (pH 7.5)/10 mM 2-mercaptoethanol/10 mM EDTA was mixed with $100 \mu \mathrm{l}$ of a $6 \mathrm{M}$ guanidine- $\mathrm{HCl}$ solution in water ( $\mathrm{pH}$ 7.0). The mixture was incubated at room temperature and $10-\mu 1$ aliquots were taken out at various times and assayed for enzymatic activity. The inset presents on a logarithmic scale the inactivation data corrected for the residual activity attributed to contamination of the assay mixture by rabbit muscle PGK (see text for details). 


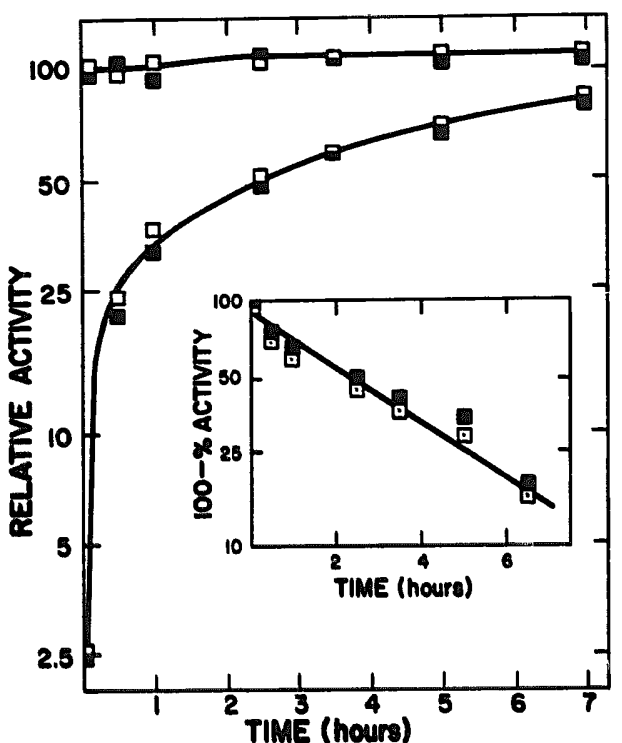

Fig. 4. Reactivation of young (D) and old () rat heart PGKs following unfolding in $2 \mathrm{M}$ guanidine- $\mathrm{HCl}$ for $16 \mathrm{~h}$ at $4^{\circ} \mathrm{C}$. The reactivation was initiated by a 10 -fold dilution of the inactivated enzyme solutions into denaturant-free buffer (100 mM sodium phosphate ( $\mathrm{pH}$ 7.5)/10 mM 2-mercaptoethanol/10 mM sodium EDTA). The final concentration of the enzymes was $30 \mu \mathrm{g} / \mathrm{ml}$ and the reactivation at $4^{\circ} \mathrm{C}$ was followed by assaying PGK activity as a function of incubation time. The upper curve presents a control experiment where samples of young and old PGKs were mixed with water (instead of guanidine- $\mathrm{HCl}$ ) and subsequently treated exactly like the unfolded samples. The activity of these controls was unaffected by the experimental procedures used. The inset presents the reactivation data of the main plot through the decline in the percentage of inactive enzyme (depicted on a logarithmic scale) as a function of incubation time in the reactivation medium.

naturant-free buffer. A typical experiment is presented in Fig. 4 and reveals a striking similarity between the refolding patterns of the young and old forms of PGK. This behavior strongly indicates the existence of a common unfolded form for both enzymes, revealing that no age-related modifications persist in the unfolded old PGK. A similar behavior was recently found by us using rat skeletal muscle PGK [17]. The implications of this observation on the nature of the age-related modification in cardiac PGK will be elaborated in the Discussion.

When the reactivation data are presented through the decline in the fraction of inactive enzyme as a function of time (Fig. 4, inset), the refolding process is seen to be governed by a first-order reaction mechanism. This refolding of rat heart PGK is different from the one observed for the skeletal muscle enzyme, since in the latter case the reactivation of the enzyme under similar conditions displayed two distinct steps [17]. The first-order reactivation kinetics reported here indicate that refolding of heart PGK either involves a transition between two states only (unfolded and inactive, folded and active) or, if intermediate states are present, that the transitions among them are relatively fast. It is interesting to note here that Betton et al. [19] found the refolding of horse muscle PGK to be biphasic; however, the fast phase was extremely rapid (less than $5 \mathrm{~ms}$ ) and would not have been detected in our measurements. When these authors monitored the refolding of PGK through the reactivation of the enzyme, a first-order mechanism was observed.

\section{Heat-inactivation of unfolded-refolded young and old PGKs}

Since the rates of heat-inactivation of young and old heart PGKs differ greatly (Fig. 1), this experimentation is a sensitive and convenient way to elucidate the status of the refolded enzymes. Heat-inactivations of refolded PGKs were performed both in Tris and in phosphate buffers. Fig. 5 depicts the inactivation patterns in each of these two buffers and clearly demonstrates the great similarity between the refolded young and old PGKs, and between these two enzyme species and native young PGK. In contrast, native old PGK is unambiguously much more heat-stable.

The native young enzyme used as the control in the heat-inactivation experiment was taken from its ammonium sulfate precipitate and dissolved in the buffer immediately before being used. This protocol was adopted since we found that samples of young enzyme incubated in solution gradually become heat-stable, and if left in solution for the length of the unfolding-refolding procedure, they resemble old PGK in their inactivation pattern (see Fig. 5A). It is important to note that this increase in heat-stability of young PGK does not involve any significant change in the enzyme's activity (as evidenced from the behavior of the control samples used in reactivation experiments - see Fig. 4) and apparently reflects in vitro aging of the enzyme leading to the production of 'aged' PGK. While the possible molecular origin of this 

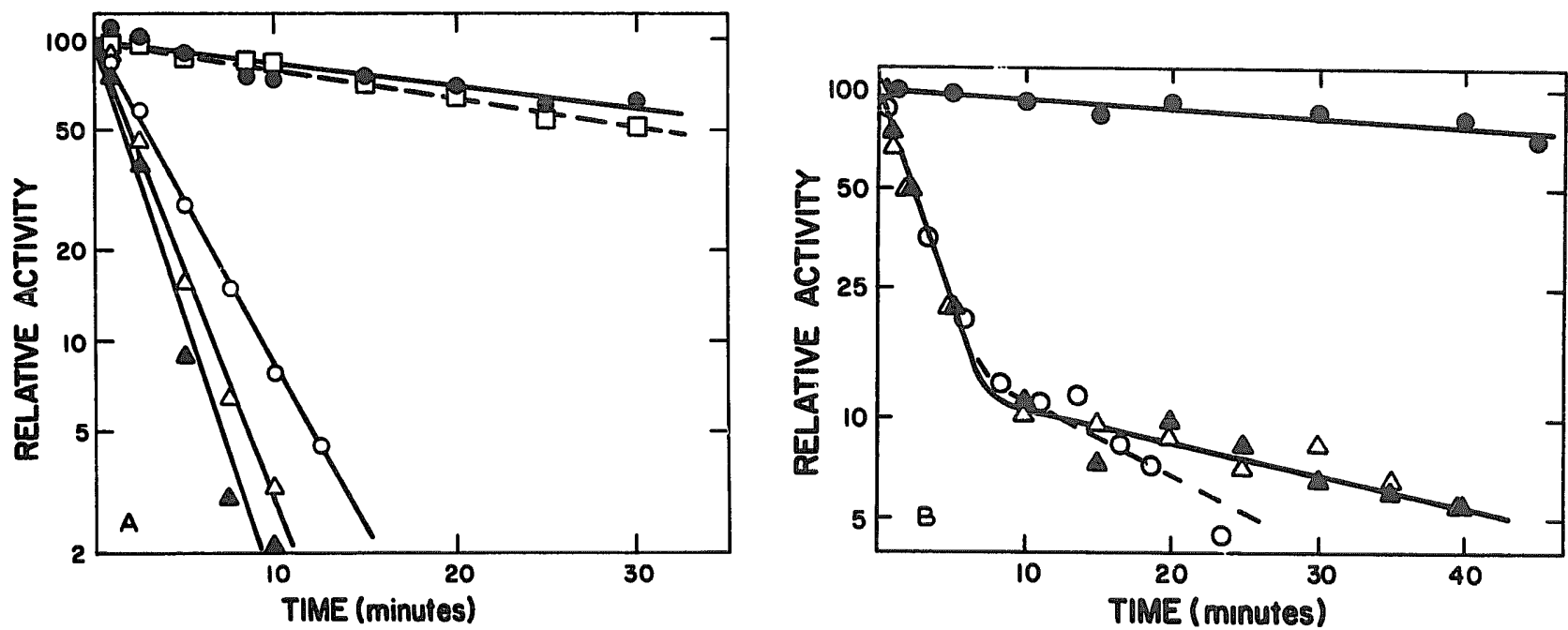

Fig. 5. Heat inactivation of native and unfolded-refolded young and old heart PGKs. (A) Inactivation at $46^{\circ} \mathrm{C}$ in $70 \mathrm{mM}$ Tris-acetate buffer (pH 7.3)/35 mM MgCl $2 / 10 \mathrm{mM}$ soaium EDTA $/ 10 \mathrm{mM}$ 2-mercapioethanol. Unfolded-reiolded young $(\Delta)$ and old ( $\Delta$ ) PGKs and their native counterparts $(O$ and $O)$ freshly dissolved from their precipitates in ammonium sulfate. The heat inactivation pattern of a sample of young PGK which has been dissolved in denaturant-free buffer and incubated at $4^{\circ} \mathrm{C}$ for the length of the unfolding-refolding procedure (about $40 \mathrm{~h}$ ) is also shown (D). (B) Heat inactivation patterns of unfolded-refolded young $(\Delta)$ old $(\Delta)$ PGKs as well as their native counterparts ( $O$ and $O$, respectively) at $50^{\circ} \mathrm{C}$ in $100 \mathrm{mM}$ sodium phosphate buffer (pH 7.5$) / 10 \mathrm{mM}$ 2-mercaptoethanol/10 mM sodium EDTA.

aging process will be discussed later, it is pertinent to note here that a similar in vitro aging was observed by us to take place in young rat muscle PGK [17]; however, the heart enzyme undergoes the modification at a much higher rate than the skeletal muscle enzyme. This correlates well with the heart PGK's greater lability in guanidine- $\mathrm{HCl}$ and its increased heat sensitivity. It is also worthwhile to note here that samples of 'aged' young PGK which have been unfolded in guanidine- $\mathrm{HCl}$ followed by refolding yield an enzyme which, when unfolded with the denaturant, displays the same unfolding kinetics as young PGK (Brown and Gafni, unpublished results).

The great similarity between the heat-inactivation patterns of the unfolded-refolded young and old PGKs in the phosphate buffer (Fig. 5B) is augmented by the fact that the inactivation under these conditions is biphasic, with an abrupt change in the reaction rate taking place at about $10 \%$ of the original activity. The two refolding products display identical inactivation profiles. Native young PGK is also seen to follow a biphasic inactivation pattern, the fast phase coinciding with that displayed by the unfolded-refolded enzymes, and similarly being responsible for about $90 \%$ of the enzyme's inactivation. The rate of the slow inactivation phase is somewhat greater for native young PGK than for the refolded enzymes. The nature of this slow inactivation phase is unclear. It may reflect the presence of $(10 \%)$ modified PGK in these samples - although it is not clear how this would be introduced into the refolded enzymes, or be due to some other, as yet unexplained, heterogeneity.

\section{Discussion}

The two major findings of the present study are (a) that rat cardiac phosphoglycerate kinase displays age-related modifications, and (b) that these modifications may be successfully abolished by enzyme unfolding-refolding, thus rejuvenating old PGK. Previous studies have reported the existence of age-related modifications in PGK in rat skeletal muscle, liver and brain tissues [13-15]. Changes in heat-stability, in the ultraviolet absorption spectrum, in the reactivity of SH groups and in the amounts of antiserum (prepared against young muscle PGK) needed to precipitate $50 \%$ of the enzymatic activity have all been observed. The latter modification is of great interest, since it provides a means of detecting the presence of altered enzymes, even in crude tissue homo- 
genates. Indeed, it was by immunotitration of such homogenates that the age-related modifications in PGK in brain and liver were first detected [13] and only later were the enzymes purified from these tissues and shown to age. In contrast, when heart (as well as kidney and lung) homogenates were tested by immunotitration, no significant age-related differences in their reactivities towards antiserum raised against young muscle PGK were found and the results were thus inconclusive. The immunotitration approach obviously relies on the assumption that the antibodies distinguish between the young and old forms of an enzyme, and while a difference in the titration profile clearly indicates the existence of age-related modifications, a lack of differences in immunoreactivity (as in the case of cardiac PGK) is no proof for the lack of such modifications and may only reflect an absence of modifications on the surface of the enzyme - which is accessible to the antibody.

Reznick et al. [20] have recently described a complementing approach where antibodies raised against denatured preparations of an enzyme were used in immunotitration of that enzyme in tissue homogenates from old animals. These antibodies were found to effectively remove inactive forms of the enzyme without affecting the active form. Antibodies raised against the active form of the enzyme, however, displayed cross-reactivity and recognized both native and modified forms of the enzyme. It is therefore not surprising that in the case of PGK - where the structural modifications in the old enzyme are apparently small, do not affect the enzymatic activity and may be localized in the interior of the molecule - antibodies raised against the young enzyme may show efficient cross-reactivity with the old form to the extent that immunotitration fails to detect age-related modifications in heart PGK. Indeed, our present study demonstrates unequivocally that rat heart PGK does become altered in old animals and, like in muscle, brain and liver enzymes, the old form of the enzyme is more heat-stable. It is interesting to speculate on the nature of the age-related modifications in PGK in the various tissues studied. Obviously, the fact that immunotitration does detect differences between young and old enzymes in some cases (i.e., muscle, brain, liver) and not in others (heart) indicates that the age-related mod- ifications in these enzymes may differ and be tissue-specific. We thus conclude from these results that, even for enzymes which exist in only one isozymic form, the different environments in various tissues may lead to the development of different modifications. As to the location of the age-related modifications, a plausible conclusion may be that in cases where immunotitration reveals an age-related difference this reflects the presence of structural changes on the protein exterior (exposed to the antibody), while in heart PGK (and possibly lung and kidney) the modifications are in sites inaccessible to the antibody.

Undoubtedly, the major finding of the present study is that the unfolded forms of young and old heart PGK are identical. This is based on the observation that the two refold with the same kinetics and that the refolding products have identical heat-inactivation rates (very similar to that of young native PGK and different from that of the old enzyme). The differences between young and old PGK thus disappear upon unfolding of the enzyme. A similar pattern was recently observed by us with PGK from rat skeletal muscle [17]. It is considered extremely unlikely that the unfolding of the old enzyme in cold $2 \mathrm{M}$ guanidine- $\mathrm{HCl}$ introduced any covalent modifications into the enzyme (such as to abolish the aging effects). We thus conclude from our data that old heart PGK (like old muscle PGK) is a conformational isomer of the young enzyme - the conformational modifications being abolished upon extensive unfolding of the enzyme. This conclusion is also supported by our observation that samples of young PGK incubated in solution, under reducing conditions, for $32 \mathrm{~h}$ resemble old PGK in their heat-stability (see Fig. 5) without any loss in activity. Here, again a covalent modification seems unlikely.

The successful rejuvenation of old rat heart PGK as described in this article provides direct evidence that the age-related modifications in heart PGK develop post-synthetically and are not, therefore, due to changes in the amino-acid sequence of the polypeptide.

Our observation that young PGK in solution is gradually transformed to a form similar to the old enzyme, with no reduction in activity, strengthens the above-mentioned conclusion and points to a possible explanation for PGK aging in vivo as 
resulting from a prolonged dwell time in the cells due to a deceleration in protein turnover in the old tissue. This hypothesis was proposed by Rothstein and co-workers $[1,12]$ and a decrease in the rate of protein turnover was indeed demonstrated to occur in Turbatrix aceti [21]. The time-scale for the development of the modifications in PGK in rat liver was evaluated by Hiremath and Rothstein [22], who found that when liver in old rats is being regenerated following hepatectomy, only the young form of PGK is produced and is gradually converted, over a period of several days, to old PGK. With heart PGK, this process may be more rapid, as we observed that, in vitro, the young enzyme was transformed to a form resembling old PGK in its heat stability within about $48 \mathrm{~h}$ of incubation in solution. In muscle PGK in vitro, we have recently found the same 'aging' process to take over 6 days [17].

The reduced stability of heart PGK as compared with the muscle enzyme (as revealed in the increased rate of heat inactivation, in guanidine$\mathrm{HCl}$ unfolding experiments, in the rate of in vitro aging) is interesting. Hiremath and Rothstein [14] found significant differences between the heat inactivation rates of muscle and liver PGKs, which are believed to be the products of the same gene [23], while brain PGK was found to be identical to the muscle enzyme [15]. Using isoelectric focusing, the latter investigators found no difference between liver, muscle and brain PGK - all three focused at $\mathrm{pH}$ 8.1. Whether skeletal muscle and heart PGK in rats are the products of different genes remains to be determined. If these two enzymes are genetically identical, then the origin of the differences observed in their properties must be in tissue-specific post-translational modifications. These modifications must be covalent, since the differences between muscle and heart PGKs are not relieved by the unfolding-refolding procedure. The nature of these differenc $s$ and the mechanism by which they develop is at present unknown and is one theme for our future work.

\section{Acknowledgements}

This work was supported by grants from the U.S. Office of Naval Research and from The
American Federation for Aging Research (to A.G.). A.Z. was the recipient of a Student Research Fellowship from the American Heart Association of Michigan.

\section{References}

1 Rothstein, M. (1982) Biochemical Approaches to Aging, Academic Press, London.

2 Rothstein, M. (1985) Mod. Aging Res. 7, 53-67.

3 Gafni, A. (1985) in Modification of Proteins During Aging (Adelman, R.C. and Dekker, E.E., eds.), pp. 19-38, Alan R. Liss, New York.

4 Gafni, A. and Noy, N. (1984) Mol. Cell. Biochem. 59, 113-129.

5 Gershon, D. (1979) Mech. Ageing Dev. 9, 189-196.

6 Schmucker, D.L. and Wang, R.K. (1983) Mech. Ageing Dev. 21, 137-156.

7 Dreyfus, J.C., Kahn, A. and Schapira, F. (1978) Curr. Topics Cell. Regul. 14, 243-297.

8 Harding, J.J. (1973) Biochem. J. 134, 995-998.

9 Gafni, A. (1981) Biochemistry 21, 6041-6046.

10 Gafni, A. (1981) J. Biol. Chem. 256, 8875-8877.

11 Reiss, U. and Rothstein, M. (1974) Biochem. Biophys. Res. Commun. 61, 1012-1016.

12 Kothstein, M. (1985) in Modification of proteins during Aging (Adelman, R.C. and Dekker, E.E., eds.), pr 53-67, Alan R. Liss, New York.

13 Sharma, H.K., Prasanna, H.R. and Rothstein, M. (1980) J. Biol. Chem. 255, 5043-5050.

14 Hiremath, L.S. and Rothstein, M. (1982) Biochim. Biophys. Acta 705, 200-209.

15 Sharma, H.K. and Rothstein, M. (1984) Mech. Ageing Dev. 25, 285-296.

16 Hardt, H. and Rothstein, M. (1985) Biochim. Biophys. Acta 831, 13-21.

17 Yuh, K.M. and Gafni, A. (1987) Proc. Natl. Acad. Sci. USA 84, 7458-7462.

18 Bradford, M. (1976) Anal. Biochem. 72, 248-254.

19 Betton, J.M., Desmadril, M., Mitraki, A. and Yon, J.M. (1985) Biochemistry 24, 4570-4577.

20 Reznick, A.Z., Dovrat, A., Rosenfelder, L., Shpund, S. and Gershon, D. (1985) in Modification of Proteins During Aging (Adelman, R.C. and Dekker, E.E., eds.), pp. 69-81, Alan R. Liss, New York.

21 Sharma, H.K., Prasanna, H.R., Lane, R.S. and Rothstein, M. (1979) Arch. Bivinem. Biophys. 194, 275-282.

22 Hiremath, L.S. and Rothstein, M. (1982) J. Gerontol. 37, 680-683.

23 Fifis, J. and Scopes, R.K. (1978) Biochem. J. 175, 311-319. 